\title{
Effect of transcranial electrotherapy stimulation on reparative regeneration of the damaged sciatic nerve in the experiment
}

\author{
Denis Dashko*, and Ivan Silkin \\ Irkutsk State Agrarian University named after A.A. Ezhevsky, Irkutsk, Russia
}

\begin{abstract}
The purpose of our research was to study the effect of transcranial electrical stimulation on the regeneration of damaged nerve fibers in laboratory animals. In the experiment, an operation was performed to cut the sciatic nerve at the level of the midthigh and to connect its ends with an epiperineural microsurgical suture. After the operation, one group (experimental) of animals was subjected to electrical exposure using electrodes inserted subcutaneously in the forehead and behind the auricles. The electric current was supplied in the mode of a combination of a direct current of $0.8 \pm 0.2 \mathrm{~mA}$ and a pulse current of $0.4 \pm 0.1 \mathrm{~mA}$ with a frequency of $70 \mathrm{~Hz}$, a pulse duration of $3.0-3.5 \mathrm{~ms}$. There were 4 sessions of exposure lasting 1 hour with an interval of three days for two weeks after the operation. The animals were monitored for 9 months. Transcranial electroanalgesia (electrostimulation) was found to activate regenerative morpho-functional processes in the nerve fibers of the damaged sciatic nerve in rats.
\end{abstract}

\section{Introduction}

The problem of analgesia affects many theoretical and practical aspects, both in medicine and in veterinary medicine [2-13]. Recently, the method of transcranial electroanalgesia (TCA) has been widely used in practice (stimulation). It has been established that electrical stimulation of certain brain structures (hypothalamus, mesencephal, medulla oblongata, etc.) can cause pronounced analgesia in animals by activating the nocic-antinociceptive system [2-13]. This system is involved not only in the regulation of pain sensitivity and the conduct of pain impulses in the central nervous system, but is also involved in trophic regulation in pathological processes in the body [16]. Therefore, the trophic effect of transcranial electrical stimulation (TCES) is fully manifested in the organs and tissues affected by the pathology, which in turn leads either to the predominance of regeneration processes, or can provoke the opposite effect - an increase in dystrophic processes at the local level.

Regeneration of peripheral nervous tissue is a complex process, in functional terms. It is assumed that endogenous opioid peptides released into the blood as a result of electrical

\footnotetext{
* Corresponding author: den120577@bk.ru
} 
stimulation of brain structures can contribute to the acceleration of regeneration processes, namely, there is a possibility of accelerating the effect of the opioid-ergic system on the nerve fibers regeneration. It follows that the possible effectiveness of the TCEA therapeutic effects is to a certain extent limited by the limits of the homeostatic capabilities of the body, which, although very large, are by no means unlimited and have significant individual differences [11]. All of the above is of particular interest in the study of the reparative capabilities of tissues, as a result of indirect exposure to them, through the central nervous system under the stimulating effect of electric currents of analgesic parameters.

The purpose of research: to study the effect of transcranial electrical stimulation on the regeneration of damaged nerve fibers in laboratory animals.

\section{Material and research methods}

The research was conducted on the basis of the Irkutsk State Agrarian University. The objects of the research were clinically healthy 50 heads of male Wistar rats weighing 200$250 \mathrm{~g}$. In 40 animals previously anesthetized with Zoletil $(1 \mathrm{mg} / 100$ grams of weight, intramuscularly), an operation was performed to cut the sciatic nerve at the level of the midthigh and to connect its ends with an epiperineural microsurgical suture $[1,14,15]$.

After the operation, one group (experimental) of animals pin the amount of 20 heads was subjected to electrical exposure using electrodes inserted subcutaneously in the forehead and behind the auricles. The electric current was supplied in the mode of a combination of a direct current of $0.8 \pm 0.2 \mathrm{~mA}$ and a pulse current of $0.4 \pm 0.1 \mathrm{~mA}$ with a frequency of $70 \mathrm{~Hz}$, a pulse duration of 3.0-3.5 ms. There were 4 sessions of exposure lasting 1 hour with an interval of three days for two weeks after the operation. The second group of 20 animals which underwent surgery was used for control.

Reinnervation of the rat's foot with efferent nerve fibers was evaluated using the vestibular motor test reflex hind limb fingers opening during a sharp lowering of the animal. The regeneration of afferent nerve fibers was studied by the method of impulse activity withdrawing from the sciatic nerve bundles during mechanical stimulation of the foot skin.

In addition, the delayed effect of transcranial electrical stimulation on the restoration of the functions of the cut and sutured sciatic nerve was studied 9 months after surgery. The functional state of the regenerating nerve was assessed by recording the compound action potential (CAP), followed by calculating the speed of nerve conduction and by recording the pulse activity of nerve fibers of the sciatic nerve during mechanical stimulation of the foot skin. The results were compared with the data obtained in 10 intact rats.

For mathematical analysis of the obtained data, the software package "Statistica" was used.

\section{Research results}

The study of the vestibular motor reflex in the animals which underwent surgery showed that the first signs of recovery of motor functions were observed in the experimental group after 5.0 1.0 days, and in the control group after $9.0 \pm 1.5$ days $(\mathrm{P} \leq 0.05)$ (Figure $1 \mathrm{~A})$. When registering the pulse activity of the nerve fibers of the sciatic nerve, it was revealed that the first impulse reactions to strong mechanical irritations of the foot skin (tingling, pricking) were observed in animals that were subjected to electrical stimulation after 14.0 1.0 , and in controls after $19.1 \pm 1.0$ days after surgery $(\mathrm{P} \leq 0.05)$ (Figure $1 \mathrm{~B})$. Transcranial electroanalgesia accelerated the reinnervation of foot skin by motor and sensitive nerve fibers, i.e. it stimulated the growth of regenerating nerve fibers. 


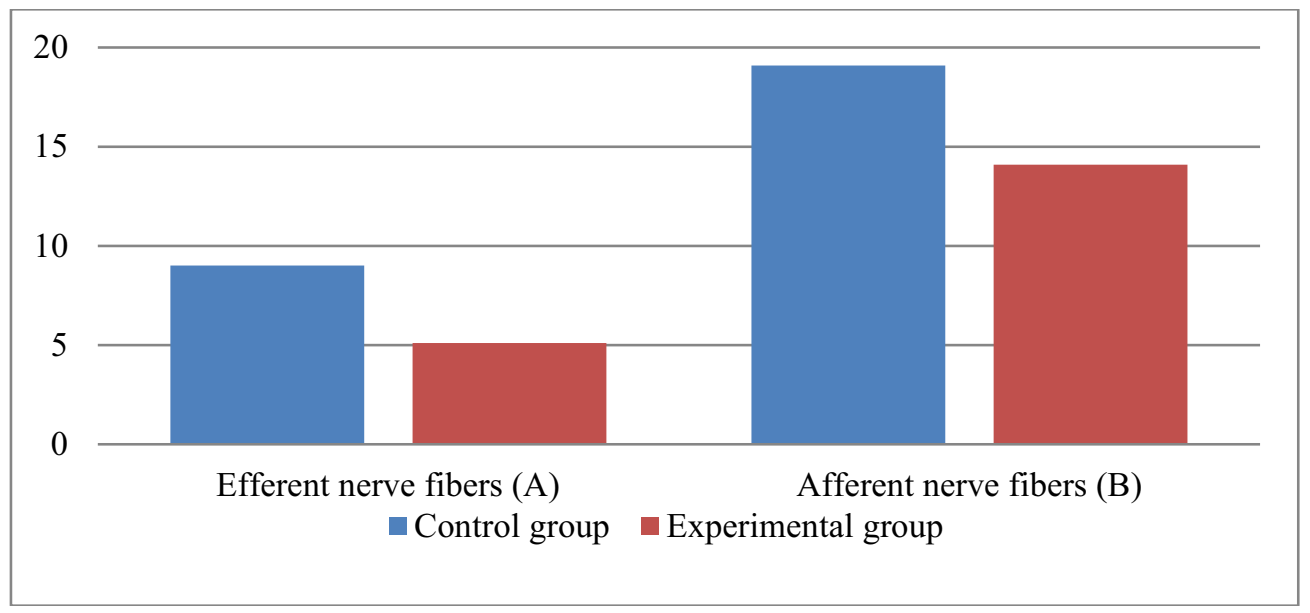

Fig. 1. Time of the beginning of foot skin reinnervation by nerve fibers of the damaged sciatic nerve, days.

Studies conducted 9 months after the operation revealed that the conduction of the sciatic nerve was completely restored in both study groups in the animals which underwent surgery (Figure 2). Nevertheless, the conduction speed along the most rapidly conducting nerve fibers, which was determined by the CAP registration method, was noticeably lower than in the norm $(37.1 \pm 0.4 \mathrm{~ms})$ and amounted to $23.1 \pm 1.0$ and $25.9 \pm 1.1 \mathrm{~ms}(\mathrm{P} \leq 0.05)$ in control and experiment, respectively.

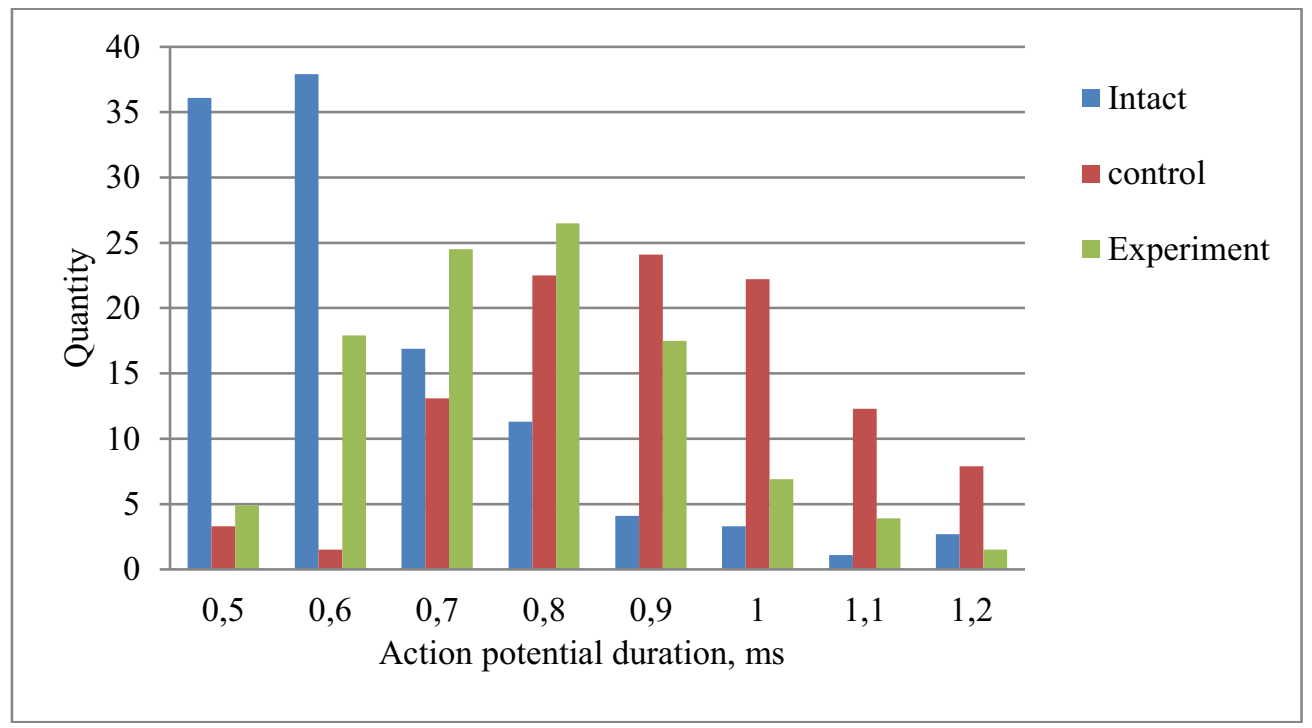

Fig. 2. Distribution of durations of the action potentials of nerve fibers in mechanical irritation of foot skin.

The analysis of the action potentials (AP) of afferent nerve fibers recorded during mechanical irritation of the foot skin showed that the AP duration in all three groups (intact, control, experimental) of animals ranges from 0.5 to $1.2 \mathrm{~ms}$. In intact animals, nerve fibers with AP duration of $0.5-0.6 \mathrm{~ms}$ predominate (the average value is $0.6 \pm 0.05 \mathrm{~ms}$ ). In control animals, 9 months after surgery, nerve fibers predominate with AP duration of $0.8-1.0 \mathrm{~ms}$, with an average value of $0.9 \pm 0.05 \mathrm{~ms}(\mathrm{P} \leq 0.05)$. In animals that underwent electrical 
stimulation, there is a significant decrease in AP duration in most nerve fibers, which brings the average value to the norm of $0.7 \pm 0.05 \mathrm{~ms}(\mathrm{P} \leq 0.05)$.

\section{Conclusion}

The results of the study showed that transcranial electrical exposure has a positive effect on regenerative processes in the peripheral nervous system. In the early stages of regeneration, electroanalgesia stimulates the growth of regenerating nerve fibers, which is manifested in the acceleration of the beginning of foot reinnervation by efferent nerve fibers, on average, by $30 \%$, and by afferent nerve fibers - by $25 \%$. A comparison of foot reinnervation terms by sensitive and motor nerve fibers revealed a slower regeneration of sensitive nerve fibers. Comparison of AP duration in intact and two groups (control, experience) of operated animals showed that transcranial electroanalgesia contributes to a better restoration of the functional properties of damaged afferents.

Thus, transcranial electroanalgesia (electrical stimulation) activates reparative morphofunctional processes in the nerve fibers of the damaged sciatic nerve in rats. The trophic effect of electrical stimulation of brain structures is fully manifested in the tissues affected by the pathology due to endogenous opioid peptides, which are released into the blood as a result of the stimulating effect of the electric current of analgesic parameters. The results of this study allow to consider the method of transcranial electrical stimulation as promising for the treatment of nerve trunk injuries and the consequences of neurosurgical operations in animals.

\section{References}

1. A.A. Aliev, Experimental surgery (Textbook, M., 1998)

2. A.V. Glotova, Experimental use of electro-anesthesia in dogs, Value of scientific student circles in the innovative development of the agro-industrial complex of the region (Publishing house of the Irkutsk SAU, 2019)

3. D.V. Dashko, V.N. Tarasevich, Relevance of the use of transcranial electrical stimulation and electro-anesthesia in veterinary practicev, Materials of the VIII International Scientific and Practical Conference "Climate, ecology, agriculture of Eurasia" (Publishing house of the Irkutsk SAU, 2019)

4. D.V. Dashko, Biophysical changes in blood in dogs with transcranial electrical analgesia, Materials of the International Scientific and Practical Conference of Young Scientists "Actual problems of biotechnology and veterinary medicine", Irkutsk, 111117 (2017)

5. D.V. Dashko, Bulletin of the IrSAA, 58, 102-108 (2013)

6. D.V. Dashko, Bulletin of the IrSAA, 57-3, 59-66 (2013).

7. D.V. Dashko, Non-traditional method of anesthesia in dogs in veterinary surgery / D.V. Dashko // Eurasian Scientific Association. 3-2 (61), 154-156 (2020)

8. D.V. Dashko, Colloquium-journal. 22-2 (46), 29-32 (2019)

9. D.V. Dashko, Determination of optimal current parameters and options for applying electrodes in electroanalgesia in dogs, Scientific research and development for implementation in the agroindustrial complex. Materials of the International Scientific and Practical Conference of Young Scientists, Irkutsk: Publishing House of the Irkutsk State Agrarian University, 183-187 (2013)

10. D.V. Dashko, Topical issues of agricultural science, 6, 27-32 (2013) 
11. D.V. Dashko, I.I. Silkin, V.N. Tarasevich, Transcranial electroanalgesia and electrical stimulation in veterinary medicine (Publishing house of the Irkutsk SAU, 144, 2020)

12. D.V. Dashko, Transcranial electroanalgesia and electrical stimulation in veterinary medicine, 56 International Scientific Conference of the Eurasian Scientific Association, Moscow 56 (3), 267-269 (2019)

13. D.V. Dashko, Experimental and clinical justification of the method of electrical analgesia in dogs (Omsk, Publishing house of the IVM OmSAU, 168, 2003)

14. A.D. Nozdrachev, E.L. Polyakov, V.A. Bagaev, Experimental surgery of laboratory animals (Textbook, St. Petersburg: Publishing house "Lan", 2007)

15. K.A. Petrakov, P.T. Salenko, S.M. Paninsky, Operative surgery with topographical anatomy of animals (M., Kolos, 2003)

16. J.G. Thalhammer et al., Anesthesiology, 82, 1013-1025 doi.org/10.1097/00000542-199504000-00026 